\title{
THE DEVELOPMENT OF MEDITERRANEAN ANAEMIA
}

\author{
BY \\ A. H. BANTON \\ From Yeovil District Hospital
}

(Received for Publication June 13, 1950)

Because of the rarity and the fact that mild forms of Mediterranean anaemia and the trait have only been recognized in recent years, opportunities for observing the development of the disease from its inception have been lacking. A recent investigation in Cyprus, where the condition is relatively common, fortuitously presented the opportunity of following two cases from birth.

The time of onset has been given as varying from shortly after birth up to the early years of life, usually the first two years. The disease may be present long before it is recognized, since increasing pallor is usually the first symptom. The presence of anaemia can, of course, be recognized by blood examination some time before pallor is noted clinically. It has also been shown that mild forms may in fact exist without the patient being aware of any disability. The case was observed during this investigation, of a young man aged 31 years with the disease in a mild form, who regularly cycled a distance of 33 miles over a rough road with no distress, is an example. He considered himself quite ilealthy.

Koch and Shapiro (1932) described a case which was recognized clinically at the age of 3 months. Fawdry (1947) diagnosed six cases by this age. Whipple and. Bradford (1932) followed a case from birth, but unfortunately no haematological examination was made until the development of pallor at the age of 8 months. I can find no evidence in the literature of any cases followed up from birth with regular blood examinations.

While examining two children suffering from Mediterranean anaemia it was noted that their mothers were again pregnant. In view of the hereditary nature of the condition it was obviously desirable to examine the offspring as soon as possible after birth. S.K. was examined three days after she was born, and I.D. 35 days after birth. Both cases were followed up at intervals and they will be considered in detail.

\section{Case Reports}

S.K. Three days after birth her haemoglobin was $12 \cdot 7 \mathrm{~g} . \%$. This is a definite anaemia considering the normal figure for that age. The film showed the characteristic features of the trait. There was a mild degree of anisocytosis and poikilocytosis; target cells were present and there was a reticulocytosis. There were no circulating normoblasts, and the changes were not as severe as would be found in the disease. At the age of 85 days circulating normoblasts were present, and the haemoglobin had fallen to $6.4 \mathrm{~g}$. \%. The mother did not as yet consider her child to be unwell, and she was quite satisfied with her progress thus far. The liver and spleen, however, were enlarged and easily palpable. Further observation at monthly intervals showed rapidly developing, severe anaemia, and the child died from fulminating bronchopneumonia at the age of 165 days. Detailed blood examinations were as follows.

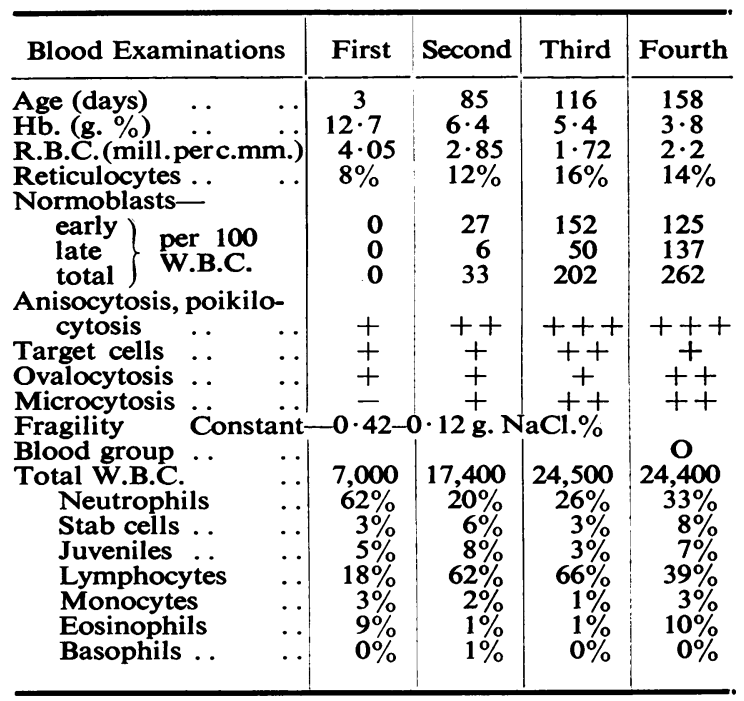

Figs. 1 and 2 show the changes in the blood films between the third and 116 th days.

I.D. This case was first examined at the age of 35 days. The haemoglobin was then 8.9 g.\%. Despite this degree of anaemia, there were no circulating normoblasts, and the film was characteristic of a severe degree 
of the trait-rather like the first film of the previous case. Though the haemoglobin had fallen to $7 \cdot 1 \mathrm{~g}$. \% by the 71 st day, the mother was still satisfied with her child's progress. Enlargement of the spleen was recognized at this stage. At 151 days the mother thought the child pale, but had no specific complaint. Detailed blood examinations were as follows.

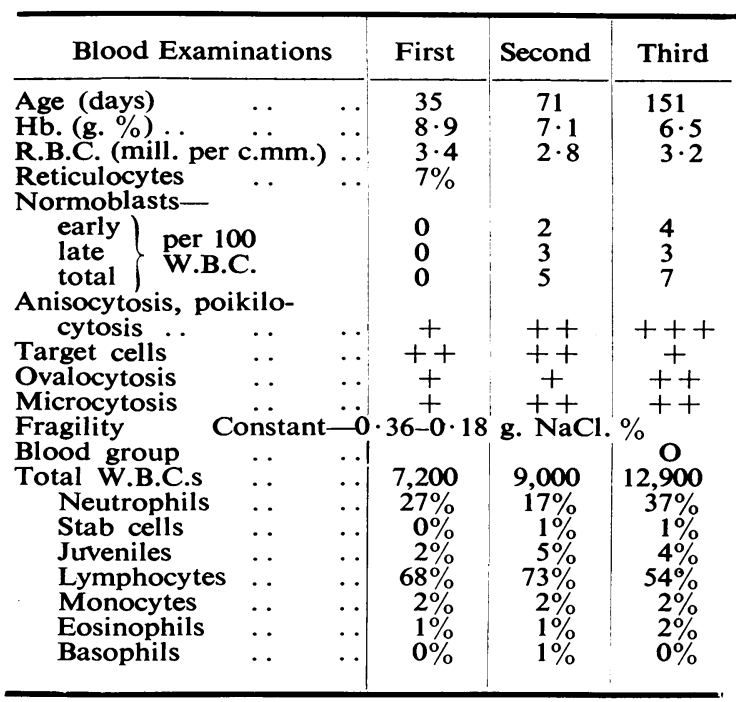

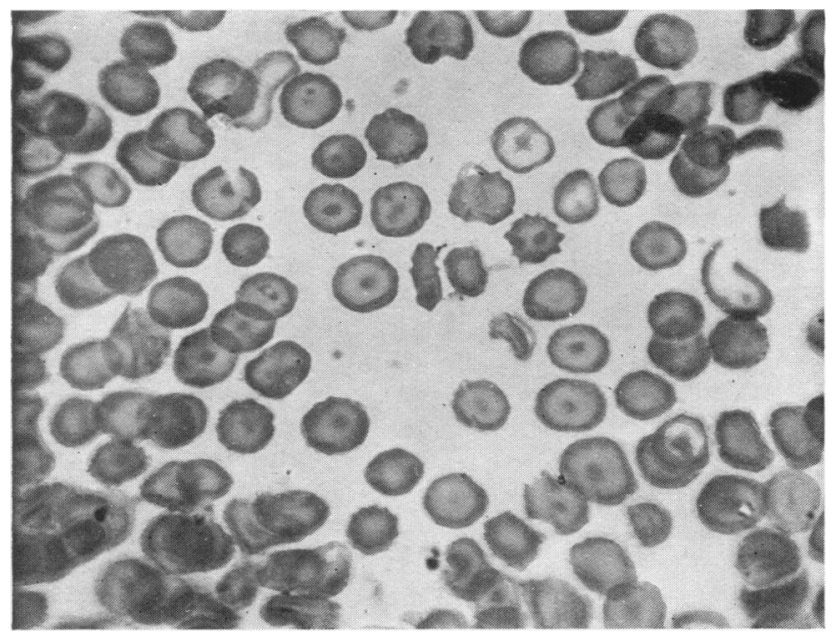

Fig. 1.-Blood film of S.K., aged 3 days. This film shows the typical features of the trait. Mild anisocytosis and poikilocytosis, target cells, and ovalocytes are seen. $(\times 850$.)

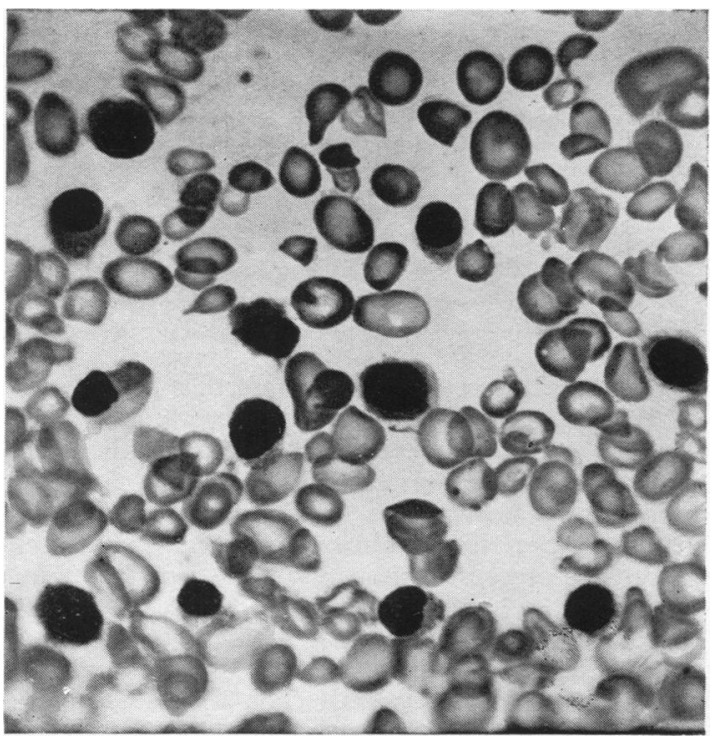

FIG. 2.-Blood film of S.K., aged 116 days. Anisocytosis and poikilocytosis have increased and normoblasts, particularly early forms, are present in the peripheral blood. There is marked hypochromia.

\section{Discussion}

The aetiology of Mediterranean anaemia has long required clarification. The development of the disease in these two cases can be correlated with recent work relating to this problem.

Though Mediterranean anaemia is a typical haemolytic anaemia, the work of Fawdry (1947) showed that there were no abnormal haemolysins in the plasma. The survival of erythrocytes from a healthy donor transfused into a case of Mediterranean anaemia was within normal limits. When blood from a case of the trait of the disease was used for the transfusion, the erythrocytes were haemolysed more rapidly than normal. This suggests that the abnormality lies in the erythrocyte rather than in the serum. Valentine and Neel (1944) point out the undesirability of transfusion of a case from one of its parents, since these are affected with the trait.

Pauling, Itano, Singer, and Wells (1949) have shown that in sickle cell anaemia an abnormal haemoglobin is present. In patients suffering from the disease all the haemoglobin is of this abnormal type, and in individuals exhibiting merely the trait of the disease only a proportion of the haemoglobin is abnormal. As is suggested (Lancet, 1950), other anaemias may be explained on these grounds and Mediterranean anaemia is clearly one of them. 
If it is accepted that the defect in Mediterranean anaemia is in the erythrocyte, possibly with an abnormal haemoglobin content, then the following hypothesis, in the light of the two cases described, may be advanced to elucidate the factors underlying the development of the disease. It is suggested that there is a defect in haemoglobin synthesis, due to the absence of some agent acting as a catalyst. Such an absence would have to be relative in order to produce that gradation in the anaemia seen between the mild and the severe cases. From a genetic point of view, since the trait is inherited as a Mendelian dominant, this variable defect would be the link with the mutated gene causing the disease, and would be the fundamental error in Mediterranean anaemia. If this is correct, one would expect the development of the disease in the first few months of life. The disease will not be fully developed at birth, since the deficient factor can be partially supplied by the mother, herself having the trait of the disease. As the source of this factor is cut off at birth, anaemia will gradually develop as the supply is used up in the early months of life. The infant's haemoglobin will fall to a level dependent on how far the infant can supply the factor from its own resources, and clearly this level will be maintained for the rest of the patient's life, except in so far as it is affected by external influences such as infections, or conditions, such as nephritis, producing anaemia, and other environmental factors. This theory will have to be tested by following other cases from birth. The age of onset judged clinically is of no value; haematological examination carried out regularly from birth is required. Carrying the theory further, cases of the trait born of mothers with the trait and normal fathers should show the trait at birth. If the father has the trait and the mother is normal, offspring who develop the trait should be normal at birth, since the normal mother can supply the deficient factor, but the trait should develop in the course of the next few months. This theory has only been elaborated since leaving
Cyprus, but it would be a simple matter to put it to the test.

Another theory to be considered is suggested by the work of Dacie and Mollison (1949). They transfused blood from a patient with nocturnal haemoglobinuria into an adult and an infant 38 days old. They showed that in both cases the transfused cells were eliminated more rapidly than normal, but the rate of elimination was considerably greater in the adult than in the infant, the significant point being that the haemolytic activity of the infant's serum was less than that of the adult. Applying this point to the problem at issue it is possible that at birth the haemolytic activity of the serum is small and hence the anaemia is not fully developed. As the haemolytic activity of the serum increases with age, so the strain on the bone marrow increases and the anaemia becomes apparent. As, however, the haemolytic activity of the serum is not likely to be materially increased in the early months of life when the anaemia is developing, this theory is discarded in favour of the former.

\section{Summary}

Two cases of Mediterranean anaemia have been observed haematologically from shortly after birth, and the development of the disease has been demonstrated in the early months of life.

Possible theories for the causation and development of Mediterranean anaemia are discussed.

\section{REFERENCES}

Dacie, J. V., and Mollison, P. L. (1949). Lancet, 1, 390. Fawdry, A. L. (1947). Annual report of D.M.S. Cyprus. Koch, L. A., and Shapiro, B. (1932). Amer. J. Dis. Child., 44, 318.

Lancet (1950), 1, 770.

Pauling, L., Itano, H. A., Singer, S. J., and Wells, I. C. (1949). Science. 110, 543.

Valentine, W. N., and Neel, J. V. (1944). Arch. intern. Med., 74, 185.

Whipple, G. H., and Bradford, W. L. (1932). Amer. J. Dis. Child., 44, 336. 